\title{
Homotypic cell membrane-cloaked biomimetic nanocarrier for the accurate photothermal-chemotherapy treatment of recurrent hepatocellular carcinoma
}

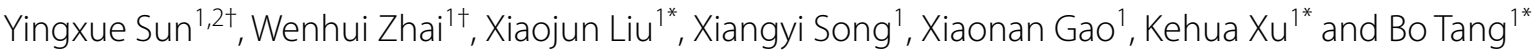

\begin{abstract}
Background: Tumor recurrence in patients after surgery severely reduces the survival rate of surgical patients. Targeting and killing recurrent tumor cells and tissues is extremely important for the cancer treatment.

Results: Herein, we designed a nano-biomimetic photothermal-controlled drug-loading platform HepM-TSL with good targeting ability and immunocompatibility for the treatment of recurrent hepatocellular carcinoma. HepM-TSL can accurately target the recurrent tumor area with the aid of the cloaked homotypic cell membrane and release the chemotherapy drugs in a controlled manner. In vivo results have confirmed that HepM-TSL loaded with drugs and photosensitizer achieves the synergistic treatment of recurrent hepatocellular carcinoma with good therapeutic effect and slight side effects.
\end{abstract}

Conclusion: Accordingly, HepM-TSL provides a sound photothermal-chemotherapy synergistic strategy for the treatment of other recurrent cancers besides of recurrent hepatocellular carcinoma.

Keywords: Homotypic cell membrane, Photothermal therapy, Chemotherapy, Synergistic therapy, Recurrent hepatocellular carcinoma

\section{Background}

Hepatocellular carcinoma (HCC), as the third leading cause of cancer-related mortality worldwide, is a common malignant tumor that seriously endangers human health [1-3]. HCC was diagnosed till advanced stages for lacking of effective therapies [4-6]. At present, partial hepatectomy is a relative curative treatment preferentially

\footnotetext{
*Correspondence: xiaojunliu@sdnu.edu.cn; xukehua@sdnu.edu.cn; tangb@sdnu.edu.cn

${ }^{\dagger}$ Yingxue Sun and Wenhui Zhai contributed equally to this work

${ }^{1}$ College of Chemistry, Chemical Engineering and Materials Science, Key Laboratory of Molecular and Nano Probes, Ministry of Education, Collaborative Innovation Center of Functionalized Probes for Chemical Imaging in Universities of Shandong, Institute of Molecular and Nano Science, Shandong Normal University, Jinan 250014, People's Republic of China

Full list of author information is available at the end of the article
}

for primary HCC patients, which can effectively treat tumor and improve the survival rate of patients $[2,7-9]$. Regrettably, $70-80 \%$ of patients undergo tumor recurrence within 5 years after surgery, greatly reducing the survival rate after surgery [10]. The high recurrence rate of $\mathrm{HCC}$ is an important issue in the treatment of liver cancer. As a result, the treatment of recurrent HCC is an urgent problem to be solved. So far, there are no current consensus guidelines to treat the patients with recurrent HCC. The current treatment methods mainly include repeat hepatectomy $(\mathrm{RH})$, radiofrequency ablation (RFA) or transarterial chemoembolization (TACE) [11-13]. In theory, the best way to treat recurrent HCC is repeated hepatectomies and liver transplantation. However, due to the practical obstacles including multicentric tumors, extrahepatic spread and inadequate normal liver reserve,

c) The Author(s) 2020. This article is licensed under a Creative Commons Attribution 4.0 International License, which permits use, sharing, adaptation, distribution and reproduction in any medium or format, as long as you give appropriate credit to the original author(s) and the source, provide a link to the Creative Commons licence, and indicate if changes were made. The images or other third party material in this article are included in the article's Creative Commons licence, unless indicated otherwise in a credit line to the material. If material is not included in the article's Creative Commons licence and your intended use is not permitted by statutory regulation or exceeds the permitted use, you will need to obtain permission directly from the copyright holder. To view a copy of this licence, visit http://creativeco mmons.org/licenses/by/4.0/. The Creative Commons Public Domain Dedication waiver (http://creativecommons.org/publicdomain/ zero/1.0/) applies to the data made available in this article, unless otherwise stated in a credit line to the data. 
repeated hepatectomies are available only for selected patients. TACE and RFA may lead to small survival benefits [14-17]. In general, the therapeutic efficacy of single therapy is still dismal.

The combination of photothermal-chemotherapy treatment brings effective therapy due to the synergistic effect $[18,19]$. Even so, problems include low targeting and low delivery efficiency of drug and photosensitizer still exist in current combination therapy [20-24]. Therefore, it is significant to design a nano-drug delivery platform that has good delivery and controlled-release effects for chemotherapy drugs and photothermal agents as well as precise target to tumor area. To this point, the use of the homotypic cancer cell membrane as the cloak of nanodrug delivery platform would be one effective strategy [25-31]. Once the nano-drug delivery platform is enveloped with the homotypic cancer cell membrane, the loaded chemotherapy drugs and photothermal agents will be released controllably in the tumor area due to the homotypic targeting ability of the cancer cell membrane, so as to improve the synergistic efficacy of photothermal therapy and chemotherapy.

Herein, in order to effectively treat the recurrent HCC through the combination of photothermal therapy and chemotherapy, we designed a drug delivery platform using homotypic cancer cell membrane as the cloak and realized the synergistic treatment of recurrent HCC with good therapeutic effect and negligible side effects. As shown in Scheme 1, the nano-drug delivery platform HepM-TSL constructed by the thermosensitive liposome (TSL) vesicles which were coated with the HCC cell membrane, and the chemotherapy drug (doxorubicin, Dox) and photosensitizer (indocyanine green, ICG) were encapsulated into HepM-TSL, noted as ICGDox-HepM-TSL. With the help of the homotypic HCC cell membrane, ICG-Dox-HepM-TSL can escape the immune system and precisely target the recurrent HCC area. The encapsulated DOX and ICG could be controllably released in tumor area when decomposition of TSL was induced by laser irradiation, and photothermalchemo synergistic therapy was achieved. The in vivo results proved that the recurrent $\mathrm{HCC}$ in the mice drastically reduced with the therapy of ICG-Dox-HepM-TSL, accompanied by slight side effects on the normal organs and tissues. In a word, nano drug delivery platform developed in this work can target the tumor area precisely, and release the drug in a controlled manner, making the combination of photothermal and chemotherapy effective, which is expected to provide a basis for the treatment of recurrent tumors.

\section{Methods \\ Preparation of TSL nanoparticles}

The blank heat-sensitive liposome nanoparticles were placed in dialysis bag (3500D) and dialyzed in secondary water. After $8 \mathrm{~h}$, the $\mathrm{pH}$ value of the internal and external aqueous phase was adjusted to make the $\mathrm{pH}$ value of the external aqueous phase 7.8. Then, the chemotherapeutic drug Dox and the photosensitizer ICG and the thermosensitive liposome nanoparticles were added to the liposome solution at a ratio of 1:1:10, and mixed evenly, then the above mixed solution were incubated in a $39^{\circ} \mathrm{C}$ water

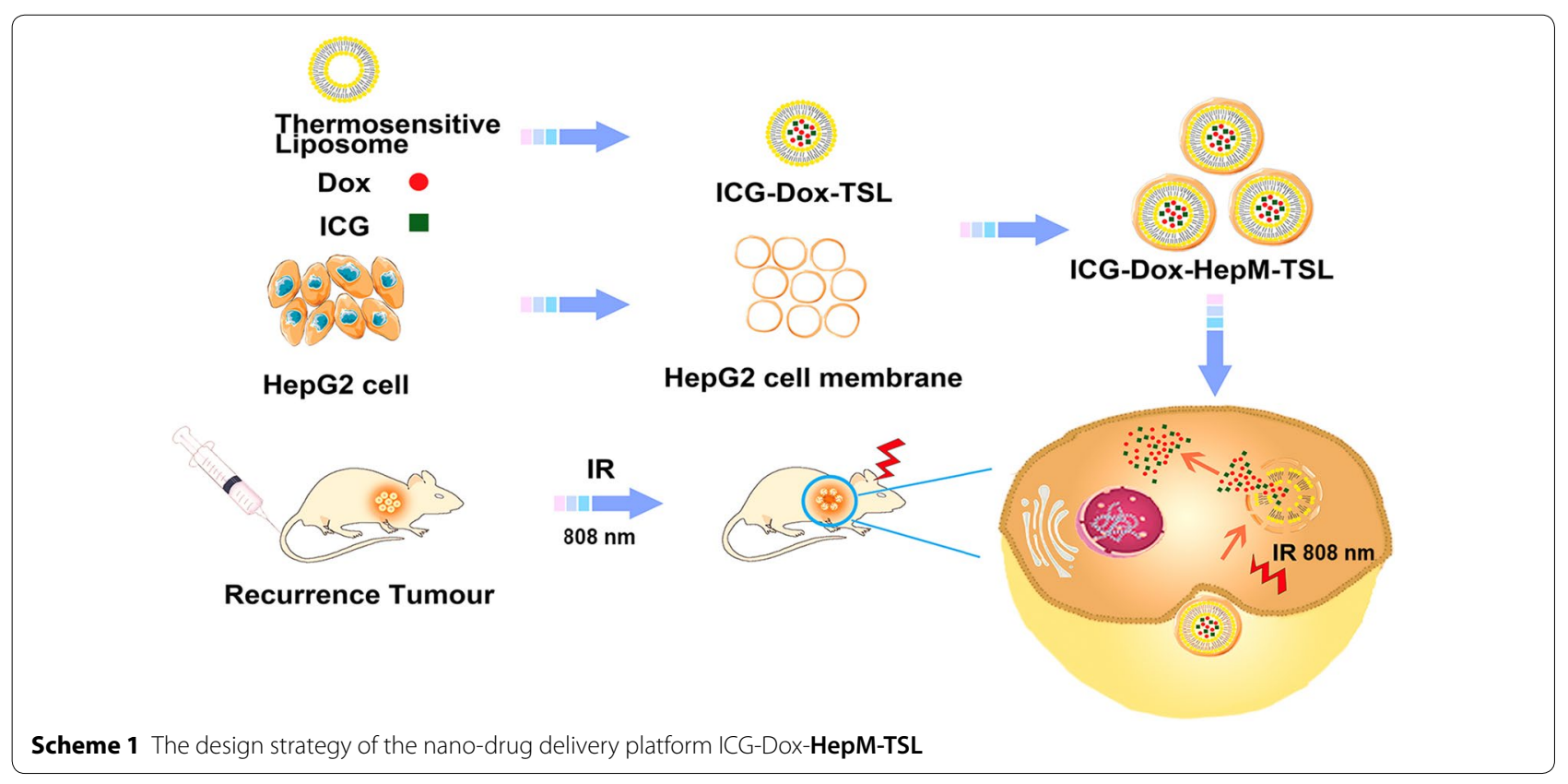


bath for $30 \mathrm{~min}$. The mixture was then placed in a dialysis bag for $24 \mathrm{~h}$ to dialyze off the free chemotherapeutic drug and photosensitizer.

\section{Preparation of cell membrane-cloaked TSL nanoparticles}

$2 \mathrm{~mL}$ of TSL nanoparticles $(1.0 \mathrm{mg} / \mathrm{mL})$ was mixed with $1 \mathrm{~mL}$ of HepG2 cell or L02 cell membrane vesicles $(0.5 \mathrm{mg} / \mathrm{mL})$. Then, the mixture was sonicated for $10 \mathrm{~min}$ $(40 \mathrm{~kW})$. Then, the cell membrane-cloaked TSL nanoparticles were collected by centrifugation treatment at $14,000 \mathrm{rpm}$ for $10 \mathrm{~min}$ at $4{ }^{\circ} \mathrm{C}$, and the supernatant was discarded. The cell membrane-cloaked TSL nanoparticles were resuspended in $3 \mathrm{~mL}$ of secondary water. The concentration of Dox in the stock liquid was $41.32 \mu \mathrm{g} / \mathrm{mL}$.

\section{Flow cytometry analysis}

Flow cytometry was used to assess the in vitro therapeutic effect of ICG-Dox-HepM-TSL. HepG2 cells were seeded and cultured for $24 \mathrm{~h}$ in $2 \mathrm{~mL}$ of DMEM $(10 \%$ FBS). After the supernatant was discarded, the HepG2 cells were incubated with ICG-Dox-HepM-TSL and ICG-Dox-TSL and free Free-ICG-Dox for $4 \mathrm{~h}$. The incubation buffer was prepared by diluting the stock solution, $24.2 \mu \mathrm{L}$ of ICG-Dox-HepM-TSL/ICG-Dox-TSL was mixed with $975.8 \mu \mathrm{L}$ of DMEM containing $10 \% \mathrm{FBS}$, and finally the concentration of Dox in the final incubation solution was $5 \mu \mathrm{g} / \mathrm{mL}$. The concentration of Dox in ICGDox-HepM-TSL, ICG-Dox-TSL nanoparticles, free-ICGDOX was kept the same. The above cells were divided into two groups, one of which was irradiated with infrared light and the other group was used as a blank control. After the incubation buffer was discarded, the cells were trypsinized (free from EDTA), collected by centrifugation at $800 \mathrm{rpm}$ for $5 \mathrm{~min}$ and washed thrice with PBS (pH 7.4). Finally, the Annexin V-FITC/PI Apoptosis Detection Kit were used to stain the above cells and an Image-StreamX multispectral imaging flow cytometer (Amnis Corporation) was used to examine the apoptosis of these cells. The flow cytometry data were analyzed using IDEAS software.

\section{In vivo tumor image}

Select 4 to 6 weeks of BALB/c nude mice weighing 15-20 g were used. The mice were housed in cages (5 per cage) and regularly fed rat chow and water. In order to build a solid tumor of HCC in nude mice subcutaneously, $5 \times 10^{6}$ HepG2 cells were injected subcutaneously into the flank region of the nude mice. When the tumor volume of the nude mice reached $100-200 \mathrm{~mm}^{3}$, Surgical removal of tumors from mice and retention of $10 \mathrm{~mm}^{3}$ tumor tissue for simulated tumor recurrence. After 1 week of recovery, the mice were randomly divided into 3 groups and the tail intravenous injected with ICG-Dox-HepM-TSL and its counterparts at regular intervals. The quality of the drug was the same in each group ( $5 \mathrm{mg} / \mathrm{kg}$ mouse). The concentration of Dox in ICG-Dox-HepM-TSL, ICG-Dox-TSL nanoparticles were kept same. Group 1 was injected with $100 \mu \mathrm{L}$ of PBS with or without near-infrared irradiation and was the control group, Group 2 was injected with ICG-Dox-TSL solution with or without near-infrared irradiation, and Group 3 was injected with ICG-Dox-HepM-TSL solution with or without near-infrared irradiation. $24 \mathrm{~h}$ after mice tail intravenous injection,they were irradiated with NIR for $5 \mathrm{~min}$.

After the above groups of mice were treated for 13 days, the nude mice were subjected to the live imager and photothermal imager and the fluorescence imaging of Dox in the nude mice were collected. Then, $24 \mathrm{~h}$ after the final injection the nude mice were sacrificed and the blood were collected by cardiac puncture. Dox fluorescence imaging was performed for ex vivo tissue from the main organs (heart, liver, spleen, lung and kidney) and tumors. H\&E staining was performed on the main organs and tumor tissues. Then the collected blood was centrifuged at $3000 \mathrm{rpm}$ for $10 \mathrm{~min}$ and the serum were collected. The serum was dripped into the 96-well plate, then follow the instructions to measure the absorbance of the alkaline phosphatase (ALP), alanine aminotransferase (ALT), aspartate aminotransferase (AST), blood urea nitrogen (BUN) and serum creatinine (Cre) with an ELISA microplate reader. The experiment was repeated three times, and the data are shown as the mean \pm SD. The body weights and tumor volumes of the mice were measured during treatment. All animal experiments were carried out according to the Principles of Laboratory Animal Care (People's Republic of China) and the Guidelines of the Animal Investigation Committee, Biology Institute of Shandong Academy of Science, China. The statistical data were analyzed using SPSS Statistics software, for deriving standard deviation, one-way ANOVA test and Bonferroni test. A p value of 0.05 was taken as the level of significance and the data were labeled with $\left(^{*}\right)$ for $\mathrm{P}<0.05$, and for $(* *)$ for $\mathrm{P}<0.01$, Each experiment was conducted in triplicate $(\mathrm{n}=3)$.

\section{Hemolysis assay}

The blood from BALB/c mice were centrifuged at $4000 \mathrm{rpm}$ for $5 \mathrm{~min}$ and the supernatant was discarded. The erythrocytes cells were washed with PBS three times until the supernatant became clear and transparent. Finally, the erythrocytes cells were resuspended in PBS and diluted to $2 \mathrm{v} / \mathrm{v} \%$. The above erythrocytes cells were respectively mixed with different concentrations of ICG-Dox-HepM-TSL, ICG-Dox-TSL, ICG, Dox, and Tween- 80 for $4 \mathrm{~h}$ at $37^{\circ} \mathrm{C}$. After $4 \mathrm{~h}$, the mixtures were 
centrifuged at $1500 \mathrm{rpm}$ for $15 \mathrm{~min}$, and then the supernatant was collected and its absorbance $\left(\mathrm{A}_{\text {sample }}\right)$ was measured at $540 \mathrm{~nm}$ with an ELISA microplate reader. Erythrocytes were incubated with $\mathrm{PBS}$ as the positive control $\left(\mathrm{A}_{100}\right)$ and deionized water as the negative control $\left(\mathrm{A}_{0}\right)$. The hemolysis rate of the experimental group was calculated as follow. The experiment was repeated three times, and the data are shown as the mean \pm SD.

$$
\text { Hemolysis rate }(\%)=\left(\mathrm{A}_{\text {sample }}-\mathrm{A}_{0}\right) /\left(\mathrm{A}_{100}-\mathrm{A}_{0}\right) \times 100 \text {. }
$$

\section{Statistical analysis}

All the statistical data were analyzed using SPSS Statistics software, for deriving standard deviation, one-way ANOVA test and Bonferroni test. A p-value of 0.05 was taken as the level of significance and the data were labeled with $(*)$ for $\mathrm{P}<0.05$, and for $(* *)$ for $\mathrm{P}<0.01$, Each experiment was conducted in triplicate $(n=3)$.

\section{Results and discussion}

\section{Preparation and characterization of HepM-TSL}

TSL nanoparticles were first synthesized and the HepMTSL nanoparticles were prepared with HepG2 cell membranes as the cloak. Cell membranes were obtained from the HepG2 cells according to previous literatures [32, 33]. TSL nanoparticles, HepM-TSL nanoparticles and HepG2 cell membrane were characterized with varied approaches. (Figure 1a, b and Additional file 1: Fig. S1). The Zeta potential the HepM-TSL changes much compared to TSL (Fig. 1c). The protein ingredient analysis of HepM-TSL was verified with gel electrophoresis (Fig. 1d), and the membrane proteins profile of HepMTSL were similar to those of HepG2 cell membrane vesicles, ensuring the intact retain of the HepG2 cell membrane during the preparation procedure. The western blot (WB) analysis results (Fig. 1e) illustrated that the main cellular adhesion molecules including galectin-1, galectin-3 and CD47 were enriched on the surface of HepM-TSL while the main intracellular nuclear marker and mitochondrial marker i.e. histone $\mathrm{H} 3$ and COXIV were little on the HepG2 cell membrane. Results in Fig. 1d, e confirmed the selective retention of membrane on the surface of HepM-TSL.

The photothermal effects of the HepM-TSL with ICG were investigated by measuring the elevated temperatures of their suspensions $\left(50 \mu \mathrm{g} \mathrm{mL}^{-1}\right)$ under $808 \mathrm{~nm}$ NIR laser irradiation $\left(1 \mathrm{~W} \mathrm{~cm}^{-2}, 1.41 \mathrm{~W} \mathrm{~cm}^{-2}, 720 \mathrm{~s}\right)$. As the power increased, the final temperature of the HepMTSL loaded with ICG was elevated to near $60{ }^{\circ} \mathrm{C}$ (the highest final temperature) (Fig. 1f), reaching the temperature requirement for heat killing of the tumor, which means that the TSL with ICG can efficiently convert NIR laser into heat. The stabilities of HepM-TSL and the TSL nanoparticles were measured using a dynamic light

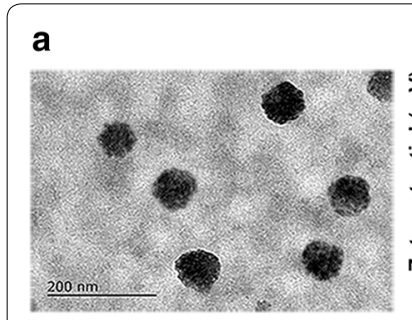

b

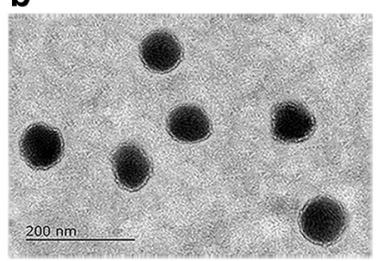



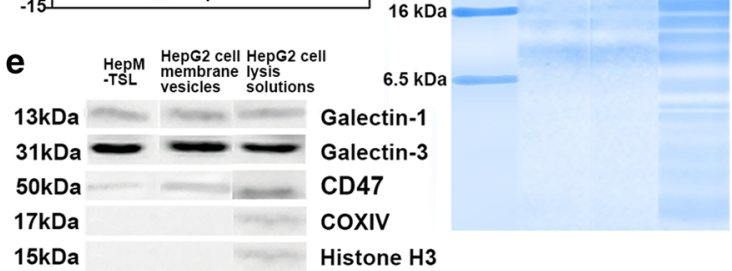

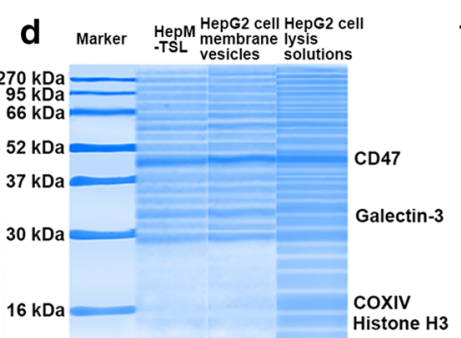

Galectin-1

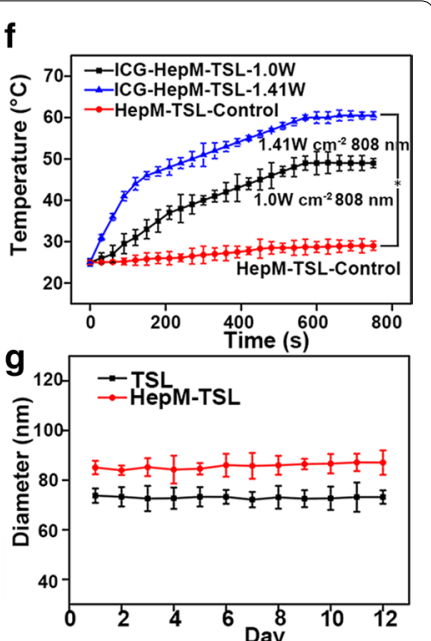

Fig. 1 Preparation and characterization of HepM-TSL. a TEM image of bare TSL nanoparticles. b TEM image of HepM-TSL nanoparticles. c Zeta potentials of bare TSL nanoparticles, HepM-TSL nanoparticles and HepG2 cell membrane vesicles. d Gel electrophoresis analysis of HepM-TSL nanoparticles, HepG2 cell membrane vesicles and HepG2 cell lysis solutions. e Western blot analysis of HepM-TSL nanoparticles, HepG2 cell membrane vesicles and HepG2 cell lysis solutions. Samples were run with equal protein concentrations and immunostained against membrane markers, including galectin-1, CD47 and galectin-3, and intracellular markers, including histone H3 (a nuclear marker), cytochrome c oxidase (COXIV,

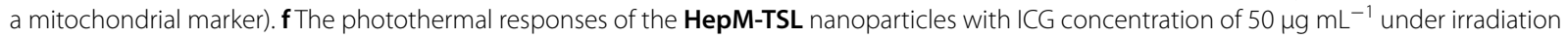
with an NIR laser $\left(808 \mathrm{~nm}, 1.0 \mathrm{~W} \mathrm{~cm}^{-2}\right.$ and $\left.1.41 \mathrm{~W} \mathrm{~cm}^{-2}\right) . \mathbf{g}$ Stability of TSL nanoparticles and HepM-TSL nanoparticles in PBS. The TSL nanoparticles and HepM-TSL nanoparticles were dispersed in PBS at room temperature then kept for 12 days. The diameters of the nanoparticles were measured with a dynamic light scattering (DLS) analyzer every day 
scattering (DLS) analyser (Fig. 1g). After 12 days, the particle size of HepM-TSL hardly changed. In summary, it was proved that the HepG2 cell membrane coated with thermosensitive liposome was prepared with excellent stability and photothermal performance.

\section{Validating the homologous targeting ability of HepM-TSL}

The targeting ability of tumor cells by ICG-Dox-HepMTSL relies on the ability of homotypic aggregation between homologous tumor cells. The study of targeting ICG-Dox-HepM-TSL to tumor cells was carried out and results were shown in Fig. 2. Hepatoma cells (HepG2 cells) and normal hepatocytes (L02 cells) were incubated with ICG-Dox-HepM-TSL, ICG-Dox-TSL nanoparticles, PBS, respectively for $4 \mathrm{~h}$ and then characterized with CLSM. The fluorescence intensity of HepG2 cells incubated with ICG-Dox-HepM-TSL was significantly stronger than that of the cells treated with ICG-Dox-TSL (Fig. 2a-c). As to L02 cells, the fluorescence intensities show little difference in the cells incubated with ICG-Dox-HepM-TSL and ICG-Dox-TSL (Fig. 2b-d). The ability to homotypic aggregation of ICGDox-HepM-TSL was verified by further experiments as shown in Additional file 1: Fig. S2 and S3. HepG2 cell, BGC-823 cell, Hela cell and MCF-7 cell were incubated with ICG-Dox-HepM-TSL for $4 \mathrm{~h}$ and then examined by the flow cytometric assay and CLSM. It was showed that ICG-Dox-HepM-TSL precisely targeted to HepG2 cells instead of other cancer cells. The above results indicate that ICG-Dox-HepM-TSL can target to HepG2 cells by virtue of the ability of homologous aggregation of $\mathrm{HCC}$ cell membranes and can target to recurrent $\mathrm{HCC}$ tumor regions.

\section{Drug release and MTT assay}

As one drug carrier platform, the drug loading capacity and the cumulative drug release efficiency of ICG-DoxHepM-TSL in the tumor area are important issues for the treatment of recurrent tumors. Herein, Dox and ICG loading contents in ICG-Dox-HepM-TSL were determined with standard curves (Additional file 1: Fig. S4) and were $41.32 \mu \mathrm{g} / \mathrm{mg}$ and $34.83 \mu \mathrm{g} / \mathrm{mg}$, respectively. Afterwards, the in vitro cumulative release profiles of Dox from ICG-Dox-HepM-TSL treated with laser were investigated. Under the irradiation of near-infrared laser, ICG converted laser into large amount of heat, making the thermosensitive liposome broken and Dox release. The cumulative drug release Dox from ICG-Dox-HepMTSL in the presence or absence of near-infrared laser $(808 \mathrm{~nm})$ were studied (Fig. 3a, b). Results authenticated

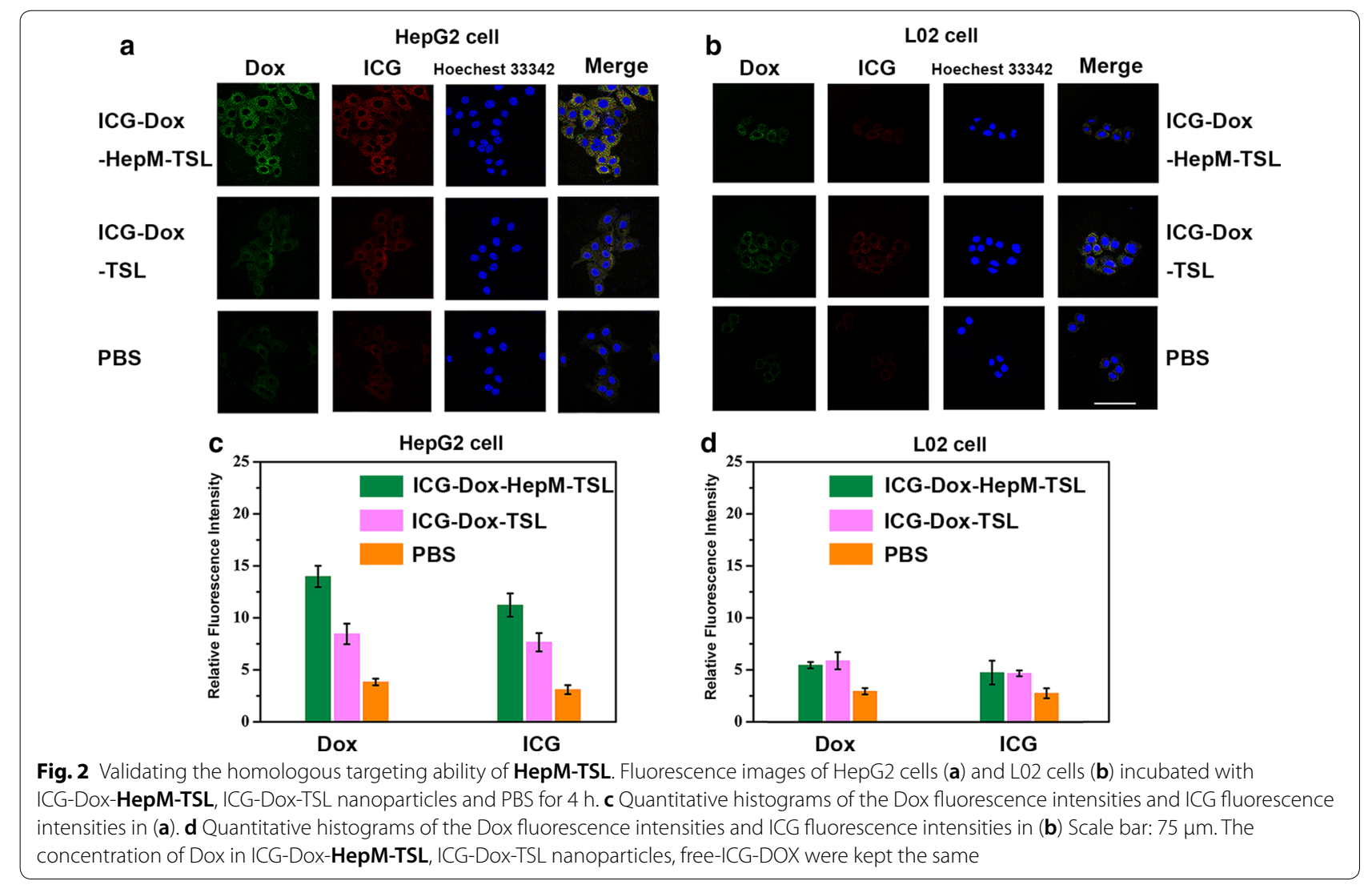



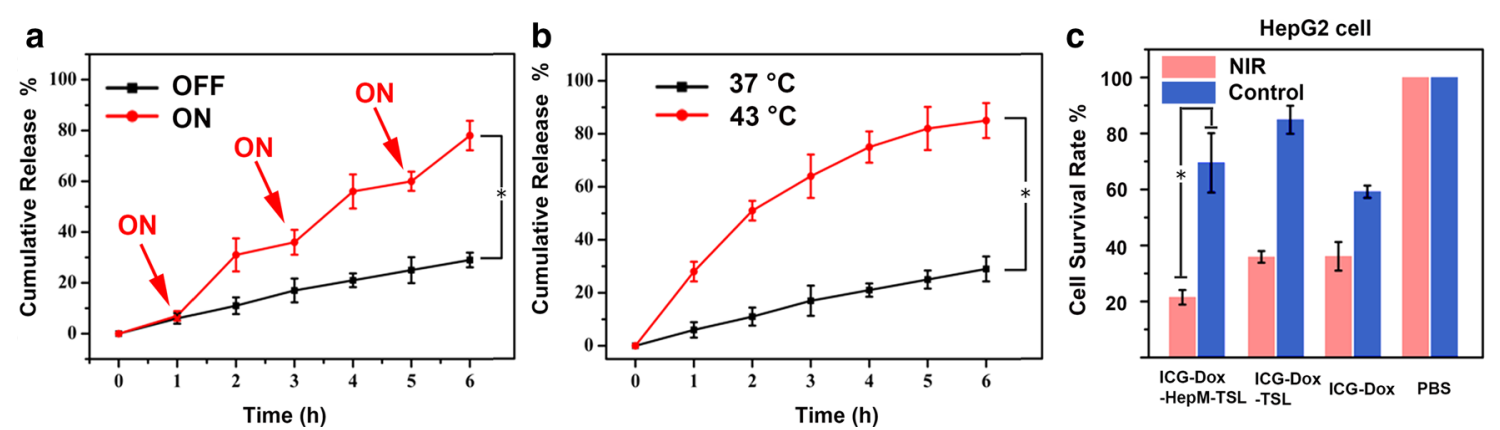

Fig. 3 Drug release and MTT assay. a The in vitro release profiles of Dox from ICG-Dox-HepM-TSL with or without of near-infrared irradiation at $37^{\circ} \mathrm{C}$. b The in vitro release profiles of Dox from ICG-Dox-HepM-TSL at $37^{\circ} \mathrm{C}$ and $43^{\circ} \mathrm{C}$ without of near-infrared irradiation. $\mathbf{c ~ M T T}$ assay results of ICG-Dox-HepM-TSL toward HepG2 cell. The concentration of Dox in ICG-Dox-HepM-TSL, ICG-Dox-TSL nanoparticles, free-ICG-DOX were kept the same

that, after three times irradiation, the cumulative release amount of DOX reached $81 \%$, largely higher than that of the control group without laser irradiation (24\%). As can be seen, the thermosensitive liposome in ICGDox-HepM-TSL made Dox controlled release by the NIR laser. The therapeutic effect of ICG-Dox-HepMTSL was evaluated by the MTT assay with HepG2 cells (Fig. 3c) Cell viability of HepG2 cells treated with ICGDox-HepM-TSL was almost $70 \%$ in the absence of NIR irradiation, but sharply decreased as to $20 \%$ after NIR irradiation. Meanwhile, under irradiation of NIR, cells treated with ICG-Dox-HepM-TSL showed much lower viability compared with those treated with ICG-Dox-TSL or free ICG-Dox. These results verified that ICG-DoxHepM-TSL had excellent photothermal-chemotherapy therapy efficiency against the recurrent HCC tumor cells.

\section{In vitro therapeutic effect}

In order to further verify the therapeutic effect of ICGDox-HepM-TSL in the treatment of cancer in vitro, HepG2 cells were incubated with ICG-Dox-HepM-TSL ICG-Dox-TSL, free ICG-Dox, PBS in presence or absence of the near-infrared laser irradiation after $4 \mathrm{~h}$. Then the
HepG2 cells were stained with Annexin V-FITC/PI and subjected to flow cytometry analysis (Fig. 4). ICG-DoxHepM-TSL strongly induced apoptosis of the HepG2 cells after exposure to near-infrared laser and the apoptosis rate of HepG2 cells was up to $52.3 \%$, (Fig. 4a, upper right quadrant, annexin $\mathrm{V}+/ \mathrm{PI}+$ ), while the HepG2 cells treated with ICG-Dox-HepM-TSL without laser irradiation or subjected to ICG-Dox-TSL were hardly in the late apoptotic stage. (Figure 4a, b, upper right quadrant). These above results indicate that ICG-Dox-HepM-TSL has excellent targeting effect on HepG2 cells, and under the illumination of near-infrared laser, both ICG and Dox could be released controllably, resulting in excellent in vitro therapeutic effect.

\section{In vivo tumor image and antitumor effect}

The in vivo experiments further proved that targeting and combination therapy of ICG-Dox-HepM-TSL were prominent (Fig. 5, Additional file 1: Fig. S5). The accumulation of ICG-Dox-HepM-TSL in the nude mice tumor sites bearing recurrent HepG2 tumor was investigated by fluorescence imaging of Dox and photoacoustic imaging 13 days after the intravenous injection with or without
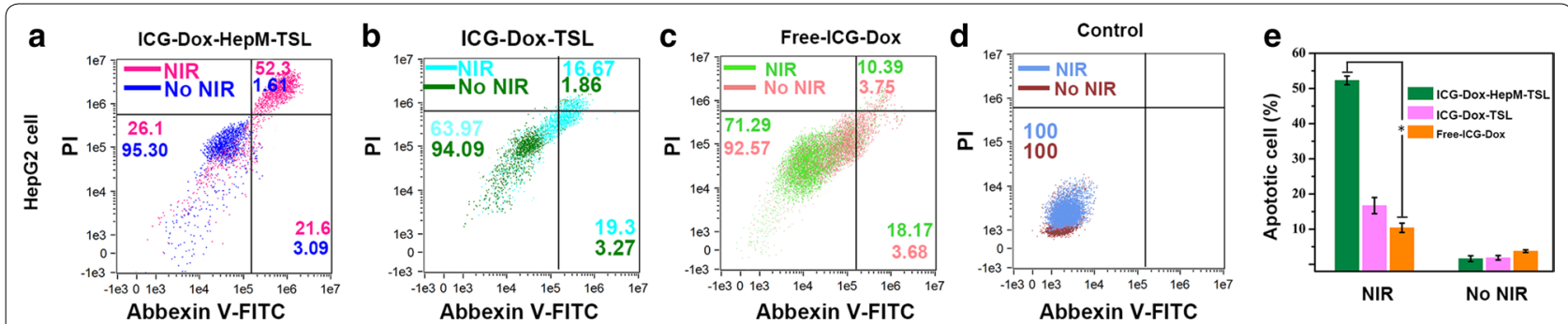

Fig. 4 In vitro therapeutic effect. a-d The flow cytometry results of HepG2 cells. HepG2 cells were stained with an Annex V-FITC/PI apoptosis kit after incubation with ICG-Dox-HepM-TSL, ICG-Dox-TSL nanoparticles, free-ICG-DOX, PBSfor 4 h. e Apoptotic cell rate of HepG2 cells in (a-d). The concentration of Dox in ICG-Dox-HepM-TSL, ICG-Dox-TSL nanoparticles, free-ICG-DOX were kept the same 


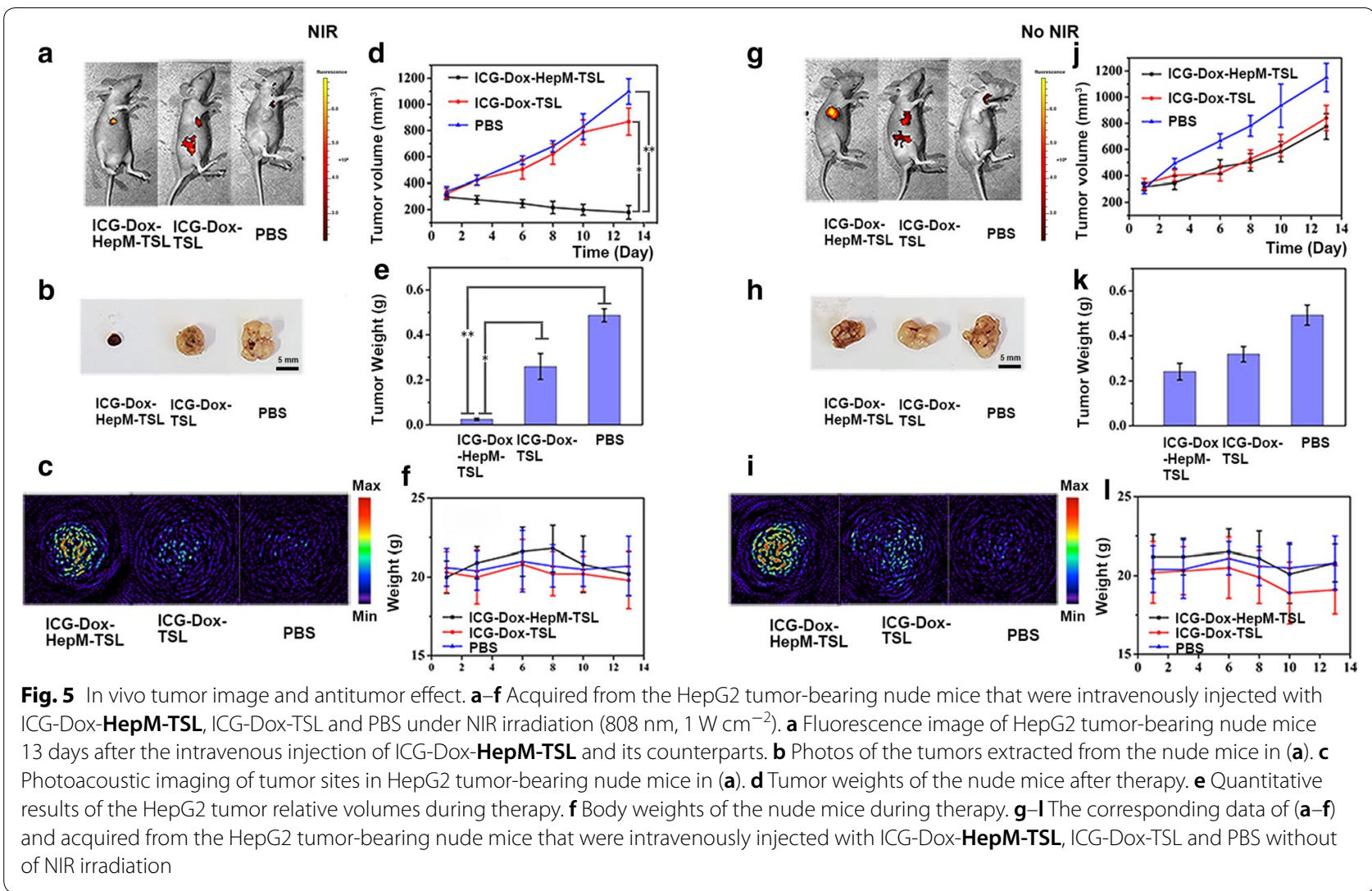

of NIR irradiation. The fluorescence intensity of Dox and photoacoustic intensity of ICG in the tumor region of mice treated with ICG-Dox-HepM-TSL under NIR irradiation were much stronger than those mice in other groups, proving the effective target ability and enrichment effect of ICG-Dox-HepM-TSL (Fig. 5a, b, g, h). The visual images of the extracted tumors and the tumor weight histograms showed the accurate photothermalchemotherapy therapeutic effect of ICG-Dox-HepMTSL on the recurrent tumor (Fig. 5c, d, i, j). Moreover, the volume of the recurrent tumor in the nude mice treated with ICG-Dox-HepM-TSL under irradiation decreased approximately $70 \%$, while obvious increase was observed in other groups (Fig. 5e-k). Further study on the damage of organs is shown in Fig. 6. Fluorescence imaging and $H \& E$ staining analysis of the main internal organs and tumor tissues of mice showed that the internal organs of mice in the ICG-Dox-HepM-TSL group were less damaged.

Hemocompatibility was examined by incubation erythrocytes with ICG-Dox-HepM-TSL, ICG-Dox-TSL, ICG, Dox and Tween 80 at gradient concentrations (Additional file 1: Fig. S6). Results indicated that, compared to Tween 80 controls (a commercial excipient intended for injectable use), ICG-Dox-HepM-TSL exhibited minimal hemolysis across all tested concentrations and had better hemocompatibility. During the clinical progression of chemotherapy drugs, the main side effects are due to their cumulative and dose-dependent hepatotoxicity and nephrotoxicity. Harsh renal side-effects significantly increased levels of blood urea nitrogen (BUN) and creatinine (Cre). The increase levels of alanine aminotransfease (ALT), aspartate transaminase (AST) and alkaline phosphatase (ALP) indicates serious hepatotoxicity. Blood biochemical indexs of ALT, AST, ALT, BUN, and Cre in plasma taken from recurrence-tumor mice were measured $24 \mathrm{~h}$ after the last injection (Fig. 7). We found that there are no significant differences between the ICGDoxHepM-TSL groups and the PBS control group in the blood biochemistry indexes (ALT, AST, ALP, BUN, Cre). Together, ICG-Dox-HepM-TSL was characterized of low toxicity, excellent biocompatibility and satisfactory therapeutic efficiency.

\section{Conclusions}

In summary, according to the homotypic aggregation and immune escape of cancer cells, we designed a nano-biomimetic photothermal-controlled drug-loading platform ICG-Dox-HepM-TSL, in which the HCC cell membrane cloaked thermosensitive liposome acted as the 

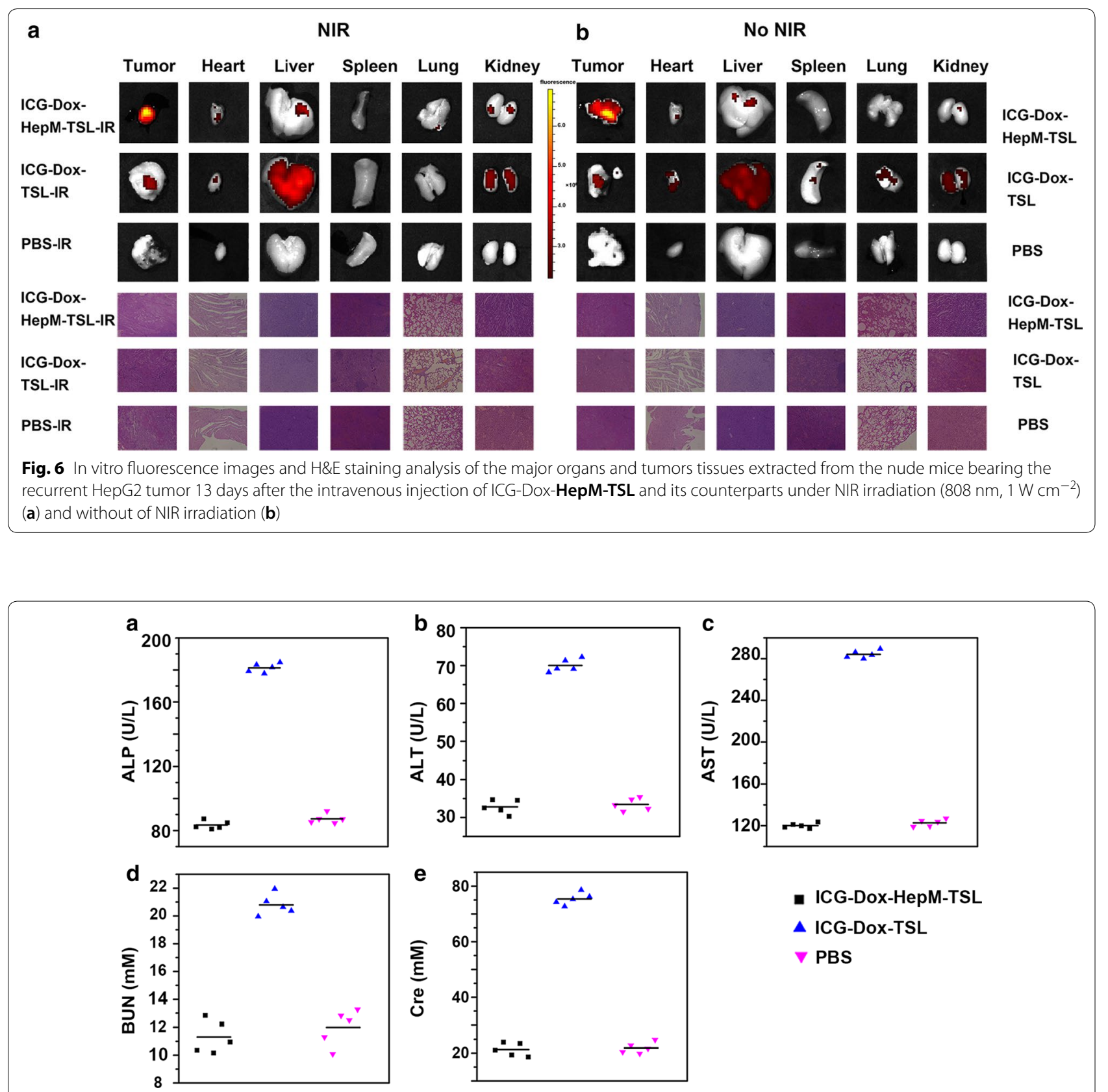

- ICG-Dox-HepM-TSL

$\triangle$ ICG-Dox-TSL

$\checkmark$ PBS

Fig. 7 Blood biochemistry data including liver-function markers: a ALP, b ALT, c AST, and kidney-function markers: $\mathbf{d}$ BUN, and e Cre. The levels in serum collected from recurrent HepG2 tumor-bearing nude mice after therapy under NIR irradiation $\left(808 \mathrm{~nm}, 1 \mathrm{~W} \mathrm{~cm}^{-2}\right.$ )

shell and the photothermal agent (ICG) and the chemotherapy drug (Dox) were cargoes. ICG-Dox-HepM-TSL could target the recurrent tumor area with the help of the homotypic HCC cell membrane. Once excited with infrared laser, ICG would generate heat; meanwhile Dox was released in a controlled manner, resulting in the synergistic effect of photothermal and chemotherapy on the recurrent HCC with little damage to normal tissues. It shows that HepM-TSL serves as a robust nanoplatform for recurrent $\mathrm{HCC}$ and provide a new strategy to the design of drug delivery platform for the treatment of cancer recurrence. 


\section{Supplementary information}

Supplementary information accompanies this paper at https://doi. org/10.1186/s12951-020-00617-2.

Additional file 1: Figure S1. TEM image of HepG2 cell membrane(a) and the ruptured liposome after the laser irradiated (b). DLS of the TSL (c), HepM-TSL (d), HepG2 cell membrane vesicles (e). Figure S2. (A) to (E) Targeting ability of HepM-TSL to HepG2 cells, BGC-823 cells, HeLa cells, and MCF-7 cell verified by flow cytometry. (F) Quantitative fluorescence intensities of results in (A) to (E). (G) Fluorescence images of HepG2 cells, BGC-823 cells, HeLa cells, and MCF-7 cell obtained with flow cytometry. Figure S3. The targeting ability of HepM-TSL to HepG2 cells and other cancer cells. (A) Fluorescence images of HepG2 cells, BGC-823 cells, HeLa cells and MCF-7 cells incubated with ICG-Dox-HepM-TSL nanoparticles. (B) Quantitative histograms of the Dox fluorescence intensities in (A). (C) Quantitative histograms of the ICG fluorescence intensities in (A). Scale bar: $75 \mu \mathrm{m}$. Figure S4. Dox and ICG loading content standard curve Dox concentration of $41.32 \mu \mathrm{g} / \mathrm{mL}$. ICG concentration of $34.83 \mu \mathrm{g} / \mathrm{mg}$. Figure S5. Schematic diagram of in vivo experiments. Figure S6. Hemolysis effect of the ICG-Dox-HepM-TSL, ICG-Dox-TSL, ICG, Dox and Tween 80.

\section{Abbreviations}

TSL: Thermosensitive liposome; Dox: Doxorubicin; ICG: Indocyanine green; HepM: Homotypic HepG2 cell membrane; ICG-Dox-HepM-TSL: Homotypic HepG2 cell membrane-cloaked thermosensitive liposome nanocarrier loaded ICG and Dox; TEM: Transmission electronic microscopy; HCC: Hepatocellular carcinoma; RH: Repeat hepatectomy; RFA: Radiofrequency ablation; TACE: Transarterial chemoembolization; WB: Western blot; NIR: Near-infrared fluorescent; MTT: 3-(4,5-dimethylthiazol-2-yl)-2,5-diphenyl tetrazolium bromide; DMEM: Dulbecco's modified eagle's medium; ALP: Alkaline phosphatase; ALT: Alanine aminotransferase; AST: Aspartate aminotransferase; BUN: Blood urea nitrogen; Cre: Serum creatinine; PBS: Phosphate buffer saline; DLS: Dynamic light scattering; COXIV: Cytochrome c oxidase IV antibody; CLSM: Confocal laser scanning microscope.

\section{Acknowledgements}

Not applicable.

\section{Authors' contributions}

YS and WZ contributed equally to this study on designing and performing the experiments. XG offered technical assistance. KX and BT were involved in the discussion. YS analyzed and interpreted the data and wrote the manuscript. XL revised the manuscript. All authors read and approved the final manuscript.

\section{Funding}

This work was supported by the National Natural Science Foundation of China (21535004, 91753111) and the Key Research and Development Program of Shandong Province (2018YFJH0502).

\section{Availability of data and materials}

All data generated or analysed during this study are included in this published article [and its Additional file 1]

\section{Ethics approval and consent to participate}

All animal experiments were carried out according to the Principles of Laboratory Animal Care (People's Republic of China) and the Guidelines of the Animal Investigation Committee, Biology Institute of Shandong Academy of Science, China.

\section{Consent for publication}

Not applicable.

\section{Competing interests}

The authors declare that they have no competing interests.

\section{Author details}

${ }^{1}$ College of Chemistry, Chemical Engineering and Materials Science, Key Laboratory of Molecular and Nano Probes, Ministry of Education, Collaborative
Innovation Center of Functionalized Probes for Chemical Imaging in Universities of Shandong, Institute of Molecular and Nano Science, Shandong Normal University, Jinan 250014, People's Republic of China. ${ }^{2}$ College of Geography and Environment, Shandong Normal University, Jinan 250014, People's Republic of China.

Received: 19 January 2020 Accepted: 9 April 2020

Published online: 16 April 2020

\section{References}

1. Jemal A, Bray F, Center MM, Ferlay J, Ward E, Forman D. Global cancer statistics. CA Cancer J Clin. 2011;61:69-90.

2. Lee EC, Kim SH, Park H, Lee SD, Lee S, Park S. Survival analysis after liver resection for hepatocellular carcinoma: a consecutive cohort of 1002 patients. J Clin Gastroenterol Hepatol. 2017;32:1055-63.

3. Erridge S, Pucher PH, Markar SR, Malietzis G, Athanasiou T, Darz A, et al. Meta-analysis of determinants of survival following treatment of recurrent hepatocellular carcinoma. Br J Surg. 2017;104:1433-42.

4. Wang FS, Fan JG, Zhang Z, Gao B, Wang HY. The global burden of liver disease: the major impact of China. Hepatology. 2014;60:2009-108.

5. Paola B, Federica T, Greta C, Teresa R, Carlo LV, Matteo M, et al. Global trends and predictions in hepatocellular carcinoma mortality. J Hepatol. 2017;67:302-9.

6. Basile N, Yaron R, Ivo D, Joseph KL. Emerging trends in hepatocellular carcinoma incidence and mortality. Hepatology. 2015;61:191-9.

7. Aysegul O. Global Epidemiology of Hepatocellular Carcinoma (HCC Epidemiology). J Gastrointest Cancer. 2017:48:2038-40.

8. Yamamoto J, Kosuge T, Takayama T, Shimada K, Yamasaki S, Ozaki H, et al. Recurrence of hepatocellular carcinoma after surgery. Br J Surg. 1996;83:1219-22.

9. Poon RTP, Fan ST, Wong J. Risk factors, prevention, and management of postoperative recurrence after resection of hepatocellular carcinoma. Ann Surg. 2000:232:10-24.

10. Bruix J, Sherman M, American Association for the Study of Liver Diseases. Management of hepatocellular carcinoma: an update. Hepatology. 2011;53:1020-2.

11. Tabrizian P, Jibara G, Shrager B, Schwartz M, Roayaie S. Recurrence of hepatocellular cancer after resection: patterns, treatments, and prognosis. Ann Surg. 2015;261:947-55.

12. Wang K, Liu G, Li J, Yan Z, Xia Y, Wan X, et al. Early intrahepatic recurrence of hepatocellular carcinoma after hepatectomy treated with re-hepatectomy, ablation or chemoembolization: a prospective cohort study. Eur J Surg Oncol. 2015;41:236-42.

13. Ho CM, Lee PH, Shau WY, Ho MC, Wu YM, Hu RH. Survival in patients with recurrent hepatocellular carcinoma after primary hepatectomy: comparative effectiveness of treatment modalities. Surgery. 2012;151:700-9.

14. Choi D, Lim HK, Rhim H, Kim YS, Yoo BC, Paik SW, et al. Percutaneous radiofrequency ablation for recurrent hepatocellular carcinoma after hepatectomy: long-term results and prognostic factors. Ann Surg Oncol. 2007; 14:2319-29.

15. Lu MD, Yin XY, Xie XY, Xu HX, Xu ZF, Liu GJ, et al. Percutaneous thermal ablation for recurrent hepatocellular carcinoma after hepatectomy. $\mathrm{Br} J$ Surg. 2005;92:1393-8.

16. Llovet JM, Bruix J. Systematic review of randomized trials for unresectable hepatocellular carcinoma: chemoembolization improves survival. Hepatology. 2003;37:429-42.

17. Llovet JM, Real MI, Montana X, Ramon P, Susana C, John A, et al. Arterial embolisation or chemoembolisation versus symptomatic treatment in patients with unresectable hepatocellular carcinoma: a randomised controlled trial. Lancet. 2002;359:1734-9.

18. Song XR, Wang XY, Yu SX, Cao JB, Li SH, Li J, et al. Co $\mathrm{Se}_{8}$ nanoplates as a new theranostic platform for photoacoustic/magnetic resonance dualmodal-imaging-guided chemo-photothermal combination therapy. Adv Mater. 2015;27:3285-91.

19. Jutaek N, Son S, Ochyl LJ, Kuai R, Schwendeman A, Moon JJ. Nat Commun. 2018:9:1074-87.

20. Hanahan D, Weinberg RA. Hallmarks of cancer: the next generation. Cell. 2011;144:646-74. 
21. Pardoll D. Does the immune system see tumors as foreign or self. Annu Rev Immunol. 2003;21:807-39.

22. Rabinovich GA, Gabrilovich D, Sotomayor EM. Immunosuppressive strategies that are mediated by tumor cells. Annu Rev Immunol. 2007;25:267-96.

23. Glinsky W, Glinsky GV, Glinskii OV, Huxley VH, Turk JR, Mossine VV, et al. Intravascular metastatic cancer cell homotypic aggregation at the sites of primary attachment to the endothelium. Cancer Res. 2003;63:3805-11.

24. Fidler IJ. The pathogenesis of cancer metastasis: the seed and soil hypothesis revisited. Nat Rev Cancer. 2003;3:453-8.

25. Kostiantyn T, Rabah B, Sabine S. Gold-graphene nanocomposites for sensing and biomedical applications. J Mater Chem B. 2015;3:4301-24.

26. Wust P, Hildebrandt B, Sreenivasa G, Rau B, Gellermann J, Riess H, et al. Hyperthermia in combined treatment of cancer. Lancet Oncol. 2002;3:487-97.

27. Luk BT, Fang RH, Hu CMJ, Copp JA, Thamphiwatana S, Dehanini D, et al. Safe and immunocompatible nanocarriers cloaked in RBC membranes for drug delivery to treat solid tumors. Theranostics. 2016;6:1004-11.

28. Gao W, Hu CJ, Fang RH, Luk BT, Su J, Zhang L. Surface functionalization of gold nanoparticles with red blood cell membranes. Adv Mater 2013;25:3549-53.
29. Luk BT, Jack HuC, Fang RH, Dehaini D, Carpenter C, Gao W, et al. Interfacial interactions between natural RBC membranes and synthetic polymeric nanoparticles. Nanoscale. 2014;6:2730-7.

30. Hu Q, Sun W, Qian C, Wang C, Bomba HN, Gu Z. Anticancer plateletmimicking nanovehicles. Adv Mater. 2015;27:7043-50.

31. Toledano FNE, Lupu-Haber Y, Bronshtein T, Kaneti L, Letko N, Weinstein E, et al. Reconstructed stem cell nanoghosts: a natural tumor targeting platform. Nano Lett. 2013;13:3248-55.

32. Chen Z, Zhao P, Luo Z, Zheng M, Tian H, Gong P, et al. Cancer cell membrane-biomimetic nanoparticles for homologous-targeting dual-modal imaging and photothermal therapy. ACS Nano. 2016;10:10049-57.

33. Xu L, Wu Sh, Wang J. Cancer cell membrane-coated nanocarriers for homologous target inhibiting the growth of hepatocellular carcinoma. J Bioact Compat Polym. 2019;34:58-71.

\section{Publisher's Note}

Springer Nature remains neutral with regard to jurisdictional claims in published maps and institutional affiliations.
Ready to submit your research? Choose BMC and benefit from:

- fast, convenient online submission

- thorough peer review by experienced researchers in your field

- rapid publication on acceptance

- support for research data, including large and complex data types

- gold Open Access which fosters wider collaboration and increased citations

- maximum visibility for your research: over $100 \mathrm{M}$ website views per year

At BMC, research is always in progress.

Learn more biomedcentral.com/submissions 